\title{
Intraocular DHODH-inhibitor PP-001 suppresses relapsing experimental uveitis and cytokine production of human lymphocytes, but not of RPE cells
}

Maria Diedrichs-Möhring ${ }^{1 \dagger}$, Sandy Niesik ${ }^{1,2 \dagger}$, Claudia S. Priglinger ${ }^{3}$, Stephan R. Thurau', Franz Obermayr ${ }^{4}$, Stefan Sperl ${ }^{4}$ and Gerhild Wildner ${ }^{1 *}$

\begin{abstract}
Background: Uveitis is a potentially blinding inflammatory disease of the inner eye with a high unmet need for new therapeutic interventions. Here, we wanted to investigate the suppressive effect of the intraocular application of the small molecule dihydroorotate dehydrogenase (DHODH)-inhibitor PP-001 on experimental relapsing rat uveitis and furthermore determine its effect on proliferation and cytokine secretion of human peripheral blood lymphocytes (PBL) and human retinal pigment epithelial (RPE) cells in vitro.

Methods: Spontaneously relapsing uveitis was induced in rats by immunization with interphotoreceptor retinoidbinding protein (IRBP) peptide R14. PP-001 was injected intravitreally after resolution of the primary disease to investigate further relapses. Proliferation and metabolic activity of phytohemagglutinin (PHA)-stimulated human peripheral lymphocytes with and without PP-001 and cytokine secretion were determined by XTT assay and bioplex bead assay. The RPE cell line ARPE-19 as well as primary human RPE cells treated with PP-001 or antivascular endothelial growth factor (VEGF) antibody bevacizumab were also investigated for metabolic activity and cytokine/chemokine secretion.
\end{abstract}

Results: Injection of PP-001 into rat eyes reduced the number of relapses by $70 \%$, from 20 relapses (57\% of the rats affected) in the control group to 6 relapses (33\% of the rats) in the treatment group. In human PBL cultures, PP-001 reduced the proliferation in a dose-dependent manner. The secretion of several cytokines such as IL-17, IFN- $\gamma$, and VEGF was suppressed by PP-001, as previously observed with rat T cells in the experimental autoimmune uveitis (EAU) model. In contrast, human RPE cells were not affected by PP-001, while the anti-VEGF antibody bevacizumab severely impaired the secretion of various cytokines including VEGF.

Conclusions: For the first time, intravitreal injection of PP-001 demonstrated an effective, but transient reduction of relapses in the rat EAU model. In vitro PP-001 suppressed proliferation and cytokine/chemokine secretion of human lymphocytes, while neither human RPE cell line ARPE-19 nor primary RPE cells were affected.

Keywords: Experimental autoimmune uveitis, Rat model, DHODH-inhibitor, Lymphocytes, RPE cells, Cytokines, Chemokines, VEGF

\footnotetext{
* Correspondence: gerhild.wildner@med.uni-muenchen.de

${ }^{\dagger}$ Equal contributors

'Section of Immunobiology, Department of Ophthalmology, University

Hospital, LMU Munich, Mathildenstr. 8, 80336 Munich, Germany

Full list of author information is available at the end of the article
} 


\section{Background}

Uveitis is a potentially blinding inflammatory disease of the inner eye. Autoimmune uveitis requires systemic immunosuppressive therapy of patients, which is frequently burdened with severe side effects. The choice of therapeutics for uveitis is modest, with corticosteroids, cyclosporine, and TNF-blocking agents as well as off-label use of methotrexate, azathioprine, and mycophenolates [1]; there is an urgent need for new and potent drugs causing less side effects. We have recently described the effect of oral administration of PP-001 (biphenyl-4-yl-carbamoylthiophene-2-carboxylic acid derivative), a new inhibitor of dihydroorotate dehydrogenase (DHODH), in two different experimental autoimmune uveitis (EAU) models in Lewis rats on inflammation as well as secondary neovascularization [2].

The two rat models of EAU are characterized by either a monophasic course of inflammation with neovascularizations as late sequel after immunization with S-Ag peptide PDSAg or a spontaneously relapsing-remitting course after immunization with IRBP peptide R14. In addition, we have shown the downregulation of proliferation and cytokine/chemokine secretion by autoreactive rat $\mathrm{T}$ cell lines. $\mathrm{T}$ cells specific for PDSAg secrete vascular endothelial growth factor (VEGF), and only PDSAginduced EAU shows chorioretinal neovascularizations $(\mathrm{CNV})$, despite a usually severe destruction of the retina in both types of uveitis and the fact that we observe $\mathrm{CNV}$ even in eyes with minor affection of the retina. We thus concluded that neovascularization in uveitis eyes is induced by the VEGF secretion of the intraocular T cells, since blocking $\mathrm{T}$ cells and their VEGF secretion by PP001 prevented CNV in this type of EAU [2].

DHODH is an enzyme necessary for pyrimidine synthesis. PP-001 inhibits DHODH with a 150-fold higher potency than teriflunomide and especially targets activating $\mathrm{T}$ cells with an enhanced requirement for pyrimidines, which can only be covered by de novo synthesis [3]. Since systemic inhibition of T cell activation leads to a generalized immunosuppression, we are aiming at an intraocular application of PP-001 to only affect the local autoimmune response while sparing systemic immunity. Here, we show the effect of intravitreally injected PP-001 solution on the course of relapsing rat EAU. Moreover, we have investigated the in vitro effect of the small molecule on human peripheral lymphocytes and different retinal pigment epithelial (RPE) cells regarding proliferation and metabolic activity as well as cytokine and chemokine production. Since VEGFproduction in PDSAg-specific, autoreactive rat $\mathrm{T}$ cells and chorioretinal neovascularizations in EAU can be suppressed by PP-001, we were interested whether PP001 could also inhibit VEGF-secretion in human RPE cells [2]. For this purpose, we have compared the effect of PP-001 and the therapeutic VEGF inhibitor bevacizumab $\left(\right.$ Avastin $\left.^{\circ}\right)$ on the human RPE cell line ARPE-19 and on primary human RPE cells, investigating cytokine and growth factor secretion. We looked at proliferation and metabolic activity and cytokine/chemokine secretion to compare it with the effect on activated human peripheral blood lymphocytes in vitro. While PP-001 efficiently downregulated proliferation and the secretion of a range of cytokines and chemokines in activated human peripheral blood lymphocytes, ARPE-19 cells and primary RPE cells were not affected. In contrast, the therapeutic VEGF inhibitor bevacizumab (Avastin ${ }^{\circ}$ ) dramatically suppressed the secretion of several cytokines by human RPE cells, including VEGF. Our data suggest that in uveitis patients PP-001 would specifically target intraocular $\mathrm{T}$ cells and their VEGF secretion and subsequent CNV induction, but without disturbing the homeostasis of basal VEGF-secretion of RPE cells that maintains the integrity of the choriocapillaris.

\section{Methods \\ Induction of EAU in rats}

Male and female Lewis rats (Lew/Orl $\mathrm{Rj}$ ) were bred in our own colony or purchased from Janvier (Le-Genest-St-Isle, France). They were maintained under specific pathogen-free conditions with water and food ad libitum and used for experiments at the age of 6-8 weeks. Animals were immunized subcutaneously into both hind legs $(50 \mu \mathrm{l} /$ side $)$ with a total volume of $100 \mu \mathrm{l}$ emulsion containing $15 \mu \mathrm{g}$ peptide R14 (human IRBP aa 1169-1191; Polypeptide Laboratories, France) and complete Freund's adjuvant (CFA), fortified with mycobacterium tuberculosis strain H37RA (BD Biosciences, Germany) to a final concentration of $2.5 \mathrm{mg} / \mathrm{ml}$. The time course of uveitis was determined by daily examination of animals with an ophthalmoscope and graded as described [4]. Clinical grading of the anterior chamber in brief, 0.5: enlargement of iris vessels, 1: peripupillar infiltration of leukocytes, 2: pupil covered with a fibrin clot, 3: anterior chamber hypopyon, and 4: anterior chamber hemorrhage. A relapse of EAU was defined as a score of $>0.5$ after the resolution of the first course of inflammation and/or a period with a score of $\leq 0.5$ or complete absence of all clinical signs of inflammation.

\section{Intraocular injection of rat eyes}

Lewis rats ( $n=30$; age $6-8$ weeks) were anesthetized using $0.5 \mathrm{mg} / \mathrm{kg}$ medetomidine s.c. Additional topical anesthesia with $0.4 \%$ oxybuprocaine eye drops was applied. After intraocular injection, sedation was antagonized with an s.c. injection of $2.5 \mathrm{mg} / \mathrm{kg}$ atipamezol. A stock solution of $35.5 \mathrm{mM}$ PP-001 (MW 479.33; 
Panoptes Pharma GmbH, Austria, corresponding to $17 \mathrm{mg} / \mathrm{ml}$ ) in absolute ethanol was diluted with sterile $0.9 \%$ saline to the final concentration of 0.5 or $0.8 \mu \mathrm{g} / \mu \mathrm{l}$. Six microliters of solution with or without PP-001 $(6.3 / 10.4 \mu \mathrm{M}$ as indicated) were injected into the vitreous of both eyes with a 30G needle, controlled under an operation microscope. The perpendicular injection is penetrating the cornea without touching the lens or the retina. After injection, an antibiotic ointment was applied to the eyes to avoid infections.

\section{Intravitreal injection of rabbit eyes and measurement of intraocular PP-001 levels}

Male Dutch belted rabbits of 13 to 18 weeks from Western Oregon Rabbit Company were used, and the experiments performed by Absorption Systems Inc. (ASI, San Diego, USA). They were housed in individual cages with water and food ad libitum. Procedures involving the care or use of animals in this study were reviewed and approved by the company's Institutional Animal Care and Use Committee (IACUC). Animals were anesthetized with an i.m. injection of ketamine hydrochloride $(30 \mathrm{mg} / \mathrm{kg})$ and xylazine $(5 \mathrm{mg} / \mathrm{kg}$ ) for the injection procedure. Topical ocular anesthetics were used. The eyes and surrounding tissues were disinfected with $2 \%$ betadine ophthalmic solution and then rinsed with saline. Using a 30G needle, $25 \mu \mathrm{l}$ PP-001 per eye was injected transsclerally 5 to $7 \mathrm{~mm}$ from the limbus. Both eyes were enucleated from animals euthanized by an intravenous injection of a commercial barbiturate-based euthanasia solution $(150 \mathrm{mg} / \mathrm{kg})$. The procedure was performed in compliance with the 2013 American Veterinary Medical Association (AVMA) Guidelines on Euthanasia. The eyes were rinsed with phosphatebuffered saline, and the retina was immediately dissected from each eye, snap frozen, and stored at $70{ }^{\circ} \mathrm{C}$ until analysis. After thawing and addition of PBS and DMSO (1:1), the samples were homogenized and sonicated. Retina samples were homogenized using a MagnaLyser (Roche). The analysis of samples is based on determination of the analytes using tandem mass spectrometry after high-performance liquid chromatography. Pharmacokinetic parameters were calculated from the time course of the tissue concentrations. Pharmacokinetic parameters were determined with Phoenix WinNonlin (v6.3) software using a noncompartmental model with sparse sampling.

\section{Human peripheral blood lymphocytes}

Collection of peripheral blood lymphocytes (PBL) from anonymized human donors with informed consent was approved by the ethics committee of the University
Hospital, LMU Munich. Lymphocytes from heparinized blood of four healthy male donors (aged 26-35) were separated by a ficoll gradient and set up as triplicate cultures in RPMI1640 without phenol red supplemented with $4 \mathrm{mM}$ L-glutamine, $1 \mathrm{mM}$ sodium pyruvate, MEM non-essential amino acids, and $50 \mathrm{U}$ penicillin $/ 50 \mathrm{mg}$ streptomycin (all from SIGMA, Deisenhofen, Germany) with or without $1 \%$ phytohemagglutinin (PHA) (Difco $\mathrm{BD}$, Heidelberg, Germany) at a density of $1 \times 10^{6} / \mathrm{ml}$ (cytokine detection in supernatants) or $2 \times 10^{6} / \mathrm{ml}$ (XTT assays). Heat-inactivated human serum pool was added to a concentration of $5 \%$. PP-001 was added to the cultures in concentrations of $3 \mu \mathrm{M}(1.44 \mu \mathrm{g}), 10 \mu \mathrm{M}$ $(4.8 \mu \mathrm{g})$, and $30 \mu \mathrm{M}(14.4 \mu \mathrm{g})$ for XTT assay and 3 and $30 \mu \mathrm{M}$ for the cytokine/chemokine detection for $72 \mathrm{~h}$. Parallel assays were set up for XTT assay and collection of supernatants. Controls included cultures without test substance but with the diluent of PP-001.

\section{Retinal pigment epithelial cell line}

The immortalized retinal pigment epithelial cell line ARPE-19 (ATTC ${ }^{\circ}$ CRL-2302 ${ }^{\mathrm{mm}}$ ) was cultured in DMEM/ Ham's F12 medium with stable glutamine supplemented with $50 \mathrm{U}$ penicillin/50 mg streptomycin and 10\%/FCS (Biochrom, Berlin, Germany). Confluent cells from passage 24 were transferred to 96-well microplates and cultured for 4 days as confluent cell layer before the medium was exchanged and PP-001 or anti-VEGF antibody bevacizumab (Roche Pharma AG, GrenzachWyhlen, Germany) were added to triplicate cultures. Controls without test substance but with the diluent of PP-001 were included. Culture supernatants were collected as described for human PBL and tested for cytokine/chemokine.

\section{Primary retinal pigment epithelial cells}

The isolation of RPE cells (RPE 80.1 and RPE 81.6) from human cadaver eyes (anonymized donors) for scientific purposes was approved by the ethics committee of the Land Oberoesterreich. Human postmortem donor eyes were obtained from the Eye Bank of the Department of Ophthalmology at the Linz General Hospital (Linz, Austria) and processed within 4-24 h after death to obtain RPE cells. Isolation of human RPE cells was performed as described previously [5]. Human RPE cells of passage 4 from two different donors were used for the experiments. Cells were maintained in DMEM/Ham's F12 (Biochrom) supplemented with 10\% FCS. For stimulation experiments, the cells were confluently seeded on 96-well plates (passage 4) 4 days before adding PP-001 or bevacizumab to triplicate cultures for $72 \mathrm{~h}$. Controls included cultures without test substance but with the diluent of PP-001. Supernatants were collected and tested for cytokines as described above. 


\section{XTT-assays}

XTT assays to determine proliferation and metabolic activity of human PBL after $72 \mathrm{~h}$ of culture were performed according to the manufacturer's instruction (R\&D Systems, Wiesbaden, Germany). Optical density (OD) was measured at $490 \mathrm{~nm}$ after $5 \mathrm{~h}$ of assay time.

\section{Bioplex bead assays to detect cytokines/chemokines}

To determine cytokine secretion and to detect early and late secreted cytokines, $25 \mu \mathrm{l}$ of supernatant was collected daily $(24,48$, and 72 h) from each well of each triplicate, pooled, and immediately frozen at $-80{ }^{\circ} \mathrm{C}$. Supernatants of triplicates from all time points ( 9 samples) were pooled immediately before testing with a Bioplex Pro Human Cytokine and Chemokine Assay (BioRad, Munich, Germany) for 29 analytes with a flow cytometry-based Luminex reader. Fifty microliters of supernatants were used for the assay according to the manufacturer's instruction and tested for IL-1 $\beta$, IL1RA, IL-2, IL-4, IL-6, IL-7, IL-8/CXCL8, IL-9, IL-10, IL12p70, IL-13, IL-15, IL-17A, Eotaxin/CCL11, basic FGF, G-CSF, GM-CSF, IFN- $\gamma$, IP-10, MCP-1/CCL2, MIP-1 $\alpha / C C L 3$, MIP-1 $\beta / C C L 4$, PDGF, RANTES/CCL5, TNF- $\alpha$, VEGF, MCP-3/CCL7, MIP-3 $\alpha / C C L 20$, and SDF $-1 \alpha+\beta /$ CXCL12. The final values obtained in the bioplex analysis are calculated from the median value of fluorescence of at least 50 measured beads per analyte and sample.

\section{Statistics}

Statistics was performed with InStat software using nonparametric (Mann-Whitney) tests (Table 1). For multiple comparison, Kruskal-Wallis test (nonparametric ANOVA) was used. $P$ values $\leq 0.05$ were regarded as significant.

\section{Results}

Intraocular injection of PP-001

Clinical course of R14-induced relapsing EAU in rats after intravitreal injection of PP-001

PP-001 or PBS was injected intravitreally in two independent experiments with a final group size of 15 rats that had R14-induced relapsing EAU. The injection was performed when the primary clinical disease course had resolved to a score of $\leqq 0.5$ (day 18 post immunization), and disease progression was observed and graded until day 31 (Fig. 1). The clinical grading only considers signs of inflammation in the anterior part of the eye, since haze (score 1) and fibrin clotting the pupil (score 2) prevent investigation of the posterior segment. One eye of the PP-001-treated group (Fig. 1, no. 7) was perforated during the injection procedure and thus excluded from the study. Two independent experiments were performed with similar results; representative time courses from the first experiment are shown in Fig. 1, and the combined data from both experiments are given in Table 1. In the first experiment $3 \mu \mathrm{g}$ PP-001 $(6.3 \mu \mathrm{M})$ was injected into each eye of the treatment group $(n=$ 10 rats, 20 eyes, Fig. 1). In the second experiment $5 \mu \mathrm{g}$ PP-001 $(10.4 \mu \mathrm{M})$ was used (5 rats, 10 eyes, time courses not shown). The rats of both treatment groups had a similar disease course prior to injection with maximal uveitis scores of 2.6 and 2.7, respectively, and a similar response to the treatment. In the PP-001-injected group, 29 eyes experienced 6 relapses, whereas in the vehicleinjected group, 20 relapses occurred in 30 eyes. Two eyes of the vehicle-injected group had 2 relapses, so 18 of 30 eyes in the control group were affected during the first 6 days post injection (days 19 to 24, Table 1). The reduction of relapses in the PP-001-treated group in relation to the control group was significant and substantial. In the control group, $73 \%(11 / 15$ rats $)$ of the

Table 1 Effect of intravitreal injection of PP-001 on R14-induced relapsing EAU in rats

\begin{tabular}{|c|c|c|}
\hline & Vehicle & PP-001 \\
\hline Mean max. clin. uveitis score primary disease (score $0-4 \pm S E$ ) & $2.6 \pm 0.1$ & $2.7 \pm 0.1$ \\
\hline $\begin{array}{l}\text { Mean max. clin. uveitis score at } \\
\text { day of injection (score } 0-4 \pm \text { SE) }\end{array}$ & $0.5 \pm 0.1$ & $0.4 \pm 0.1$ \\
\hline Total no. of relapses post injection until day 6 & 20 & 6 \\
\hline No. of eyes with multiple relapses & 2 & 0 \\
\hline No. of eyes with relapses & 18 out of 30 eyes $(60 \%)^{*}$ & 6 out of 29 eyes $(21 \%)^{*}$ \\
\hline Relapses/animals & $11 / 15(73 \%)^{*}$ & $5 / 15(33 \%)^{*}$ \\
\hline Mean max. uveitis score/relapse (score $0-4 \pm S E$ ) & $1.1 \pm 0.1$ & $1.2 \pm 0.3$ \\
\hline Mean duration of relapse (days \pm SE) & $1.45 \pm 0.11$ & $1.17 \pm 0.17$ \\
\hline Mean appearance of first relapse (days post injection \pm SE) & $3.4 \pm 0.5$ & $4.3 \pm 0.9$ \\
\hline Mean severity of uveitis post injection until day $6( \pm$ SE) & $2.22 \pm 0.25^{*}$ & $1.34 \pm 0.22^{*}$ \\
\hline
\end{tabular}

"Mean severity of uveitis" is the mean of daily uveitis scores calculated over a certain period of time. One eye of the PP-001-treated group (rat 7/PP-001, see Fig. 1) was perforated during injection and not considered. Data are pooled from two independent experiments, Exp. $1, n=10 ;$ Exp. $2, n=5$ rats/group. ${ }^{*} p<0.05$ 


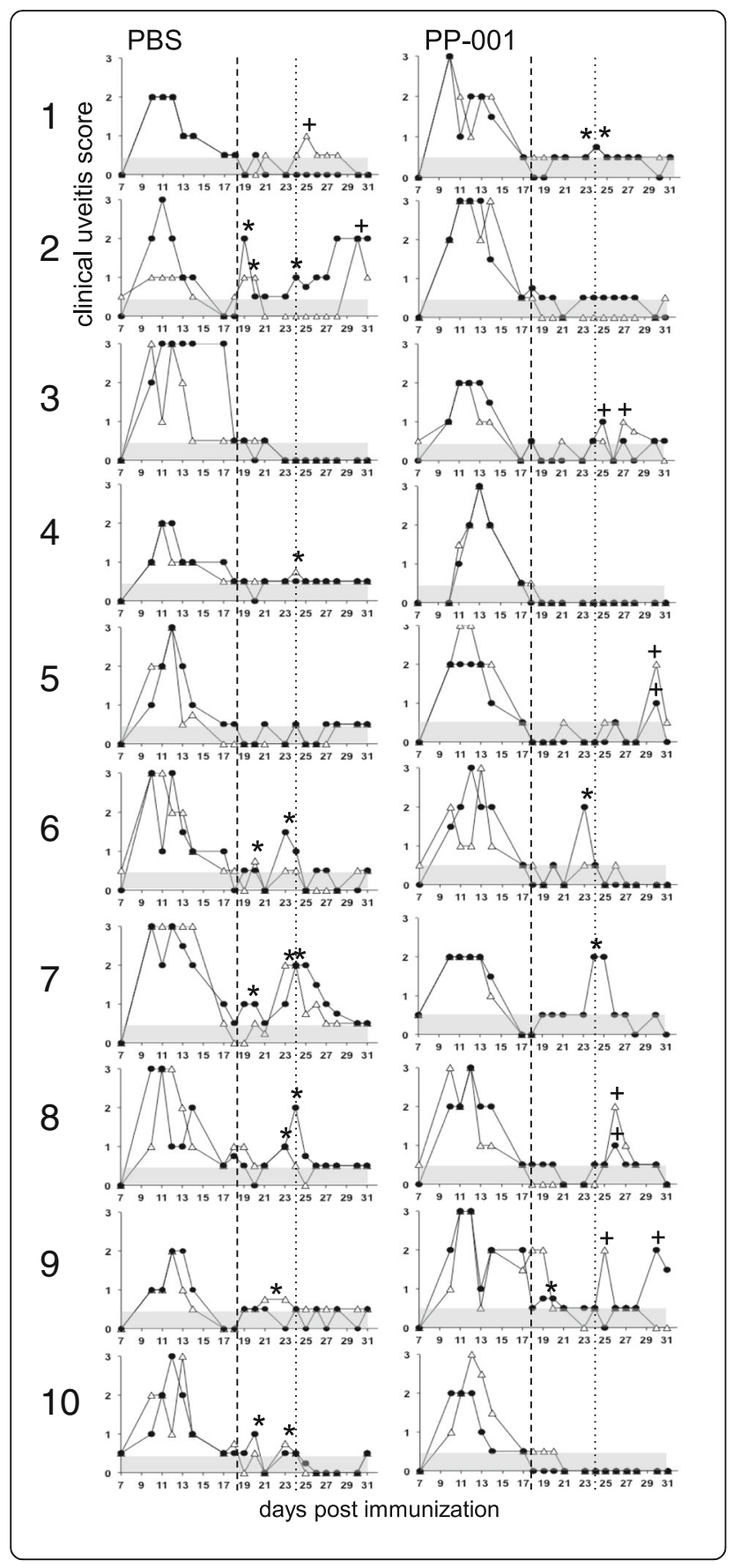

animals had relapses, but only $33 \%$ (5/15 rats) in the PP001-treated group. The number of eyes and the number of animals with relapses were significantly reduced in the PP-001-treated compared to the vehicle-treated group $(p<0.05)$, also the average daily intensity of inflammation from days 19 to $24(p<0.05)$ (PP-001, 1.34 \pm 0.22 ; vehicle, $2.22 \pm 0.25)$. The mean number of relapses/rat was 0.733 (CI 0.480-0.987) in the PBS-treated group and 0.33 (CI 0.063-0.604) in the PP-001-treated group; the mean number of relapses/eye was 0.633 (CI
Fig. 1 1-10 Clinical course of R14-induced relapsing EAU in rats after intravitreal injection of PP-001. Both rat eyes were injected once intravitreally with $6 \mu \mathrm{l}$ PBS or $3 \mu \mathrm{g}$ PP-001/6 $\mu \mathrm{l}$ PBS at day 18 (dashed line, resolution of primary inflammation) post immunization with R14CFA. The clinical course is shown for each eye from each rat of both groups. The dotted line at day 24 defines the time period of therapeutic activity of intraocular PP-001. Relapses were defined as a clinical score of $>0.5$ following a score $\leqq 0.5 .{ }^{*}$ Indicate relapses between days 19 and $24,+$ the relapses from day 25 until the end of the experiment. The gray areas show the negative/low inflammation scores that have to be exceeded for the definition of a relapse. Triangles: right eyes, black dots: left eyes. The right eye of rat PP-001/no. 7 was perforated during the injection and thus omitted from further evaluation. $N=10$ rats per group; PBS $n=20$ eyes, PP-001 $n=19$ eyes after injection. Representative data are shown from experiment 1 of two independent experiments

0.365-0.902) in the control group and 0.241 (CI $0.076-$ $0.407)$ in the PP-001 group. The average daily intensity of inflammation is shown as "severity of uveitis over time" and was calculated by the summation of the daily uveitis scores of all eyes in one group divided by the number of eyes and number of days (Table 1). PP-001 showed a significant transient suppressive effect on the relapses in R14-induced relapsing EAU. The duration of the effect is supported by a pharmacokinetic analysis of the retina after intravitreal injection (Fig. 2). It even suggests that a single local supply of efficacious dose levels of PP-001 for 24 to $48 \mathrm{~h}$ is sufficient to show an effect on relapses of EAU for 6 days.

\section{Pharmacokinetic of PP-001 in rabbit eyes}

To further investigate the fate of PP-001 within the eye, pharmacokinetic studies of PP-001 in different ocular tissues were performed after intravitreal injection of $62.5 \mu \mathrm{g}$ PP-001 into the eyes of Dutch belted rabbits. During the first $4 \mathrm{~h}$ post injection, the retinal concentration of PP-001 was stable between 83 and $98 \mu \mathrm{g} / \mathrm{g}$ retina and dropped thereafter (Fig. 2). A terminal half-life $\left(t_{1 / 2}\right)$ of $13.6 \mathrm{~h}$ was determined. After $48 \mathrm{~h}$, the PP-001 concentration in the retina was still $3 \mu \mathrm{M}$, a concentration that was still highly suppressive for human lymphocytes, as shown in Figs. 3 and 4. PP-001 levels were comparable in choroid and in vitreous humor at all time points and AUC (area under the curve; which is integral in a plot of drug concentration in blood plasma vs. time) was $1 / 3$ of that of the retina. After intravitreal injection of $62.5 \mu \mathrm{g}$ PP-001, plasma concentrations were very low $(\mathrm{Cmax}=41 \mathrm{ng} / \mathrm{ml})$. Plasma levels were $>2000$-fold lower than respective retina levels (maximal level $40.9 \mathrm{ng} \mathrm{PP}-001 / \mathrm{ml}$ ), and potential systemic antiinflammatory activity of PP-001 can be neglected at such low plasma concentrations. We have refrained from testing PP-001 levels in the inflamed eyes, since it has been published that there are no major 


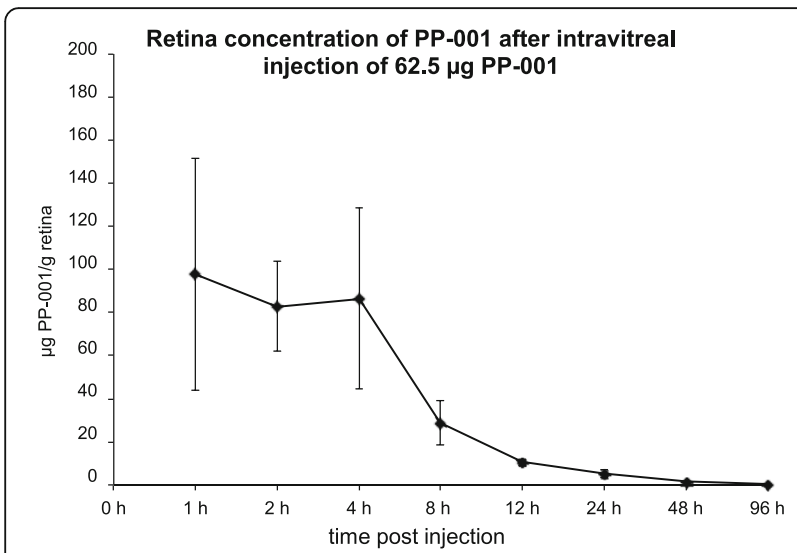

Fig. 2 Pharmacokinetic profiles of PP-001 in rabbit eyes. Retinal concentration of PP-001 after intravitreal injection of $62.5 \mu \mathrm{g} \mathrm{PP-001/}$ rabbit eye $(0 \mathrm{~h})$. Tissues were collected at the indicated time points; PP-001 concentration is shown as nanogram per milligram/retina. $N=2$ rabbits/4 eyes for each time point. Mean concentrations of PP-001 \pm SD

differences expected in the pharmacokinetics between healthy and diseased eyes [6].

\section{Effect of PP-001 on human lymphocytes and retinal pigment epithelial cells}

We have previously shown that PP-001 efficiently reduced proliferation and metabolism as well as cytokine secretion of rat $\mathrm{T}$ cells in vitro, and one of the key cytokines affected by PP-001 was VEGF in PDSAg-specific T cells [2]. Thus, we investigated whether PP-001 would have similar effects on human lymphocytes and on retinal pigment epithelial cells.

\section{XTT assay to determine metabolic activity}

Metabolic activity and proliferation of human PHAstimulated PBL were significantly suppressed in a dosedependent manner by PP-001 as determined by XTT assay (Fig. 3a), while no effect was observed on human RPE cells ARPE-19 (Fig. 3b). Figure 3a shows the mean percentage of inhibition of PHA-stimulated PBL from four donors (the same as those assayed for cytokine secretion in Fig. 4) with increasing concentrations of PP-001.

\section{Cytokine secretion}

Cytokine secretion was measured in culture supernatants from PBL stimulated with PHA and treated with PP-001 in doses of 3 and $30 \mu \mathrm{M}$. Only data from those cytokines and chemokines with a level above the detection threshold of the assay from the four donors are shown in Fig. 4. Since the tested donor lymphocytes showed similar pattern in response to PP-001, but highly varied in the amounts of secreted factors, we have calculated the mean percentage of alteration of cytokine secretion compared to PHA stimulation only, the latter was defined as $100 \%$. The highest amounts of secreted

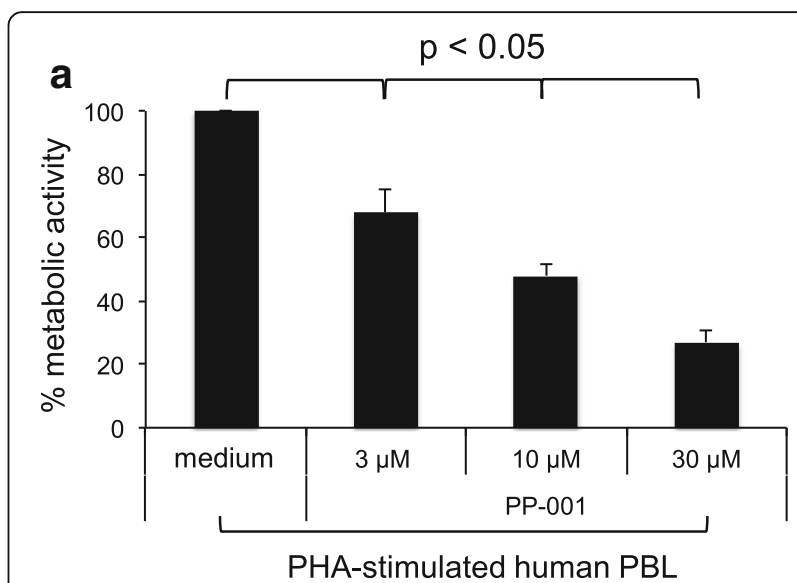

b

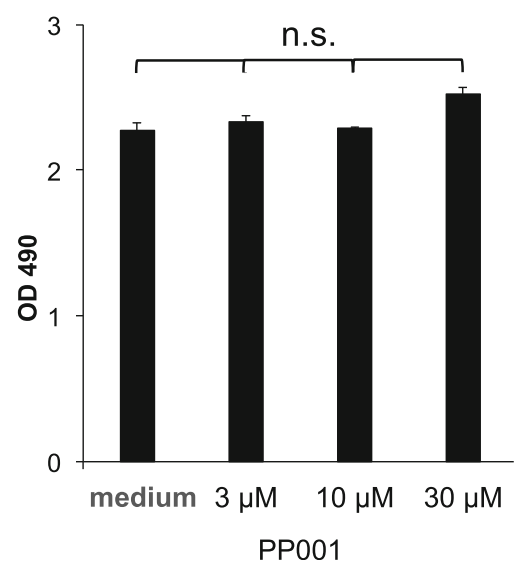

Fig. 3 Proliferation and survival (metabolic activity) of PHA-stimulated human PBL and ARPE-19 cells treated with PP-001. a Inhibition of PHA-stimulated human peripheral blood cells from four different donors with PP-001, determined by XTT assay. OD 490 was measured after $72 \mathrm{~h}$ of PBL culture in triplicates with medium only, PHA and PHA with different concentrations of PP-001. Mean medium control values were subtracted from the mean values of stimulated cultures of each individual donor. Data of cultures stimulated with PHA only were defined as 100\%; the percentage of activity (OD 490) is calculated in relation to the PHA control for each donor. Data show the means of $\%$ OD490 + SE from all donors. $\mathbf{b}$ XTT assay of triplicate cultures of ARPE19 cells being confluent for 4 days and treated with either PP-001 or medium only. OD 490 was determined after $72 \mathrm{~h}$ of culture. Data are shown as mean OD $490+$ SD

cytokines from PHA-stimulated PBL were determined for IFN- $\gamma$ and TNF- $\alpha$, followed by IL-6, IL-13, IL-2, and GM-CSF. The secretion of IL-8 from PBL is not shown, since it exceeded the detection level of the assay in all PHA-stimulated cultures. IL-17 secretion was moderate and even lower than IL-10, MIP-1a/CCL3, and VEGF. The lowest concentration of PP-001 $(3 \mu \mathrm{M})$ already efficiently and significantly suppressed the secretion of the T cell-produced cytokines IL-2, IFN- $\gamma$, IL-17, IL-13, IL-10, TNF- $\alpha$, VEGF, and GM-CSF, while the secretion of mainly monocyte-produced interleukins (monokines) IL-1 $\beta$, IL-1ra, MCP-1/CCL2, MCP-3/CCL7, MIP-1 $\beta$ / 


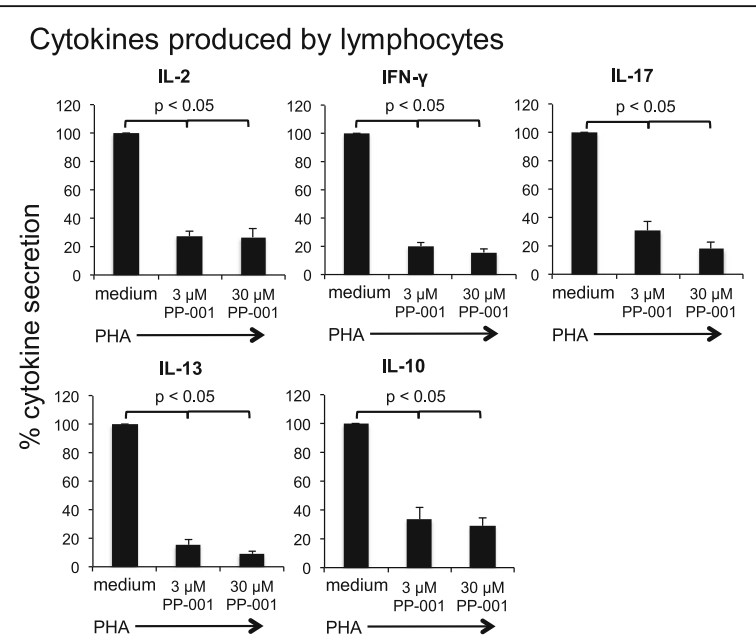

Cytokines and chemokines

produced by lymphocytes and monocytes/macrophages
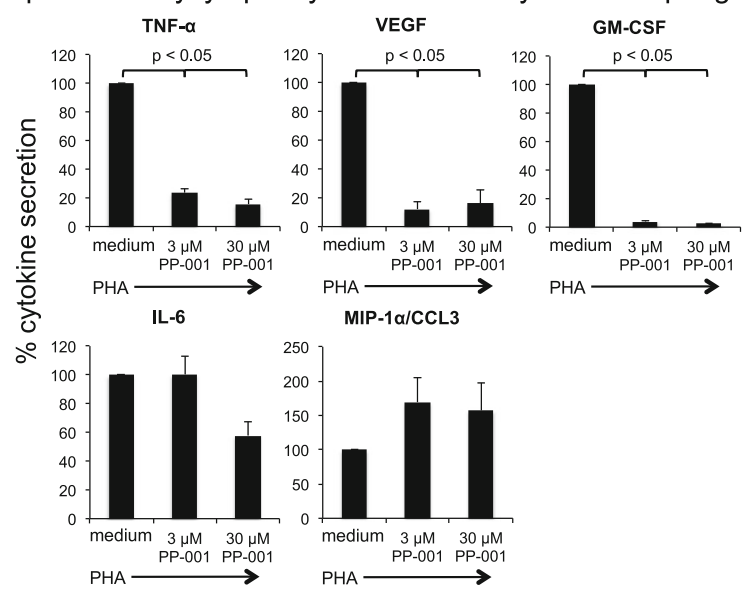

Monokines and chemokines produced by monocytes/macrophages
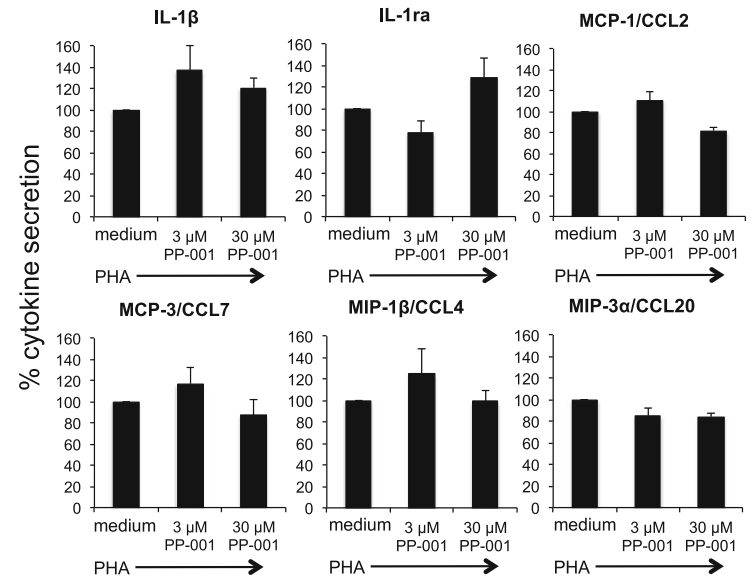

Fig. 4 Cytokine secretion of PHA-stimulated human PBL treated with PP-001. Cytokine secretion of PHA-stimulated human PBL from four donors with or without PP-001 (3 and $30 \mu \mathrm{M}$, respectively). Supernatants from triplicate cultures were collected at three time points $(24,48$, and $72 \mathrm{~h}$ after onset of cultures, in total nine samples) and pooled for the final bioplex analysis. Cytokine secretion of cultures with PHA only is defined as 100\%; the percentage of secretion in cultures with PP-001 is respectively calculated in relation to the PHA-control for each donor. Data show the means of $\%$ secretion from four different donors $+\mathrm{SE}, p$ values are only shown for significantly different data

CCL4, and MIP-3 $\alpha / C C L 20$ was not suppressed by PP001, suggesting that the $\mathrm{T}$ cell response, but not the innate immune response, was inhibited by treatment with PP-001. IL- 6 and MIP-1 $\alpha / C C L 3$ are produced by both T cells and myeloid cells and were not (MIP-1 $\alpha /$ CCL3) or only slightly inhibited with $30 \mu \mathrm{M}$ PP-001 (IL-6), suggesting that innate cells might have compensated for the suppressed $\mathrm{T}$ cell secretion of these factors (Fig. 4).

Human RPE cells were also co-cultured with various doses of PP-001 and bevacizumab as indicated (Fig. 5), and culture supernatant was collected daily to determine secreted cytokines, chemokines, and growth factors. As described for the human PBL above, only those cytokines/chemokines produced in amounts within the detection levels of the bioplex assay are shown in Fig. 5.

ARPE-19 cells and the primary human RPE cells secreted a number of cytokines, chemokines, and VEGF as the predominant growth factor. ARPE-19 cells had a higher baseline secretion of all factors compared to primary RPE cells from passage 4, except for IL-6, which was secreted in higher amounts by the primary RPE 80.1 cells (Fig. 5).

While PP-001 had almost no effect on the secretion of the tested factors and only slightly reduced the secretion of VEGF from ARPE-19 cells, bevacizumab efficiently suppressed IFN- $\gamma$, IL-10, IL-12, and IL-13 as well as VEGF secretion of ARPE-19 cells and the primary human RPE cells 80.1. IFN- $\gamma$, expressed at low levels in primary RPE, was only slightly diminished by bevacizumab, while the rather low IL-13 secretion was suppressed in RPE 80.1, but not RPE 81.6.

IL-8 production was enhanced in a dose-dependent manner with both, PP-001 and bevacizumab in ARPE19, but not in primary RPE cells. IL-6, MCP-1/CCL2, and IP-10/CXCL10 secretion was not affected in any of the cells with both PP-001 and bevacizumab.

Despite the strong suppressive effect of PP-001 on lymphocytes, RPE cells remained unaffected. In contrast, bevacizumab, which is used for intraocular injections in case of uveitis neovascularizations, suppressed cytokine secretion of RPE cells. Bevacizumab thus might influence the function and role of RPE cells in the ocular immune privilege and their interaction with neighboring ocular structures like the choriocapillaris and choroid. 


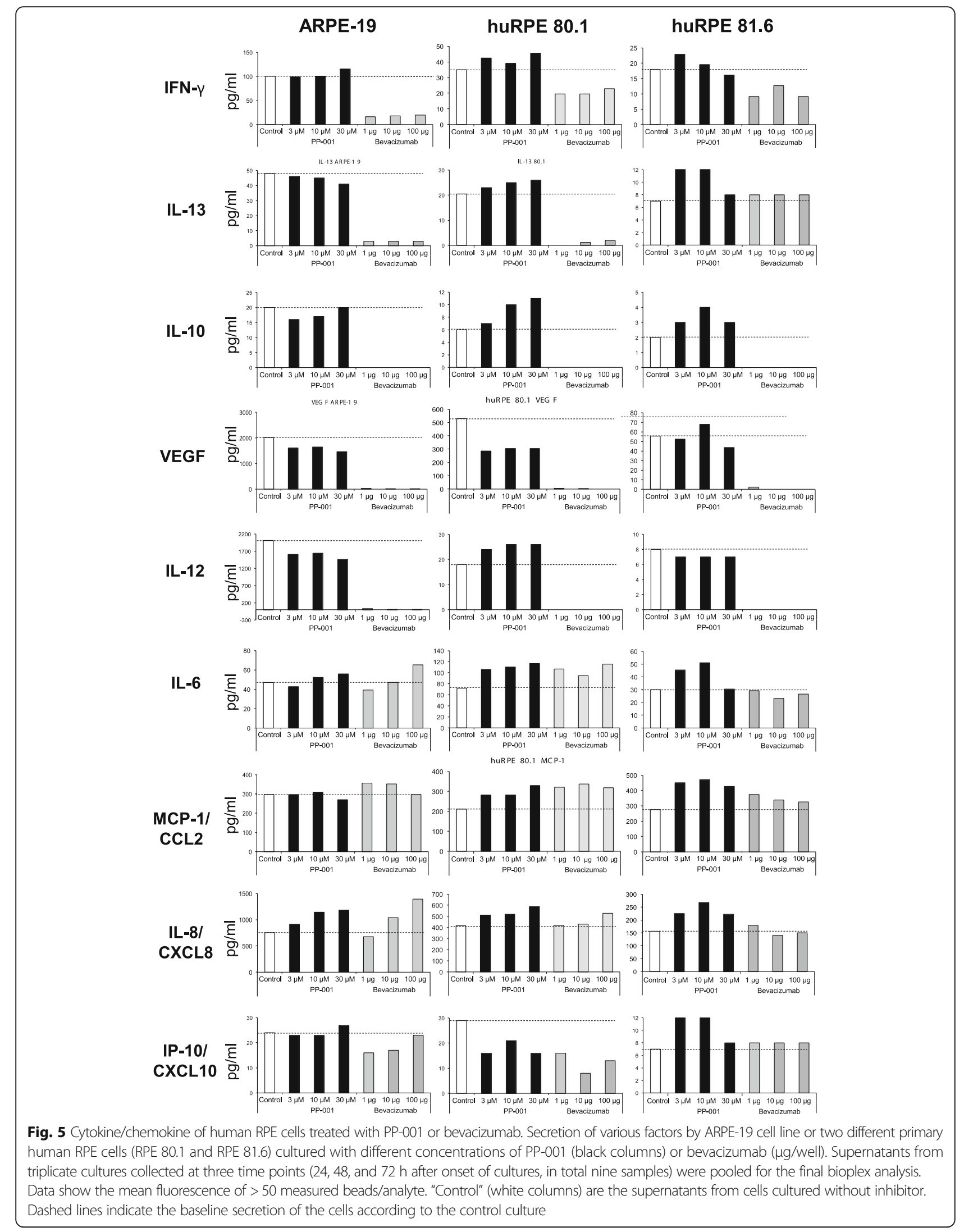




\section{Discussion}

Autoimmune uveitis is usually treated with systemic immunosuppressive agents, especially when the posterior part of the eye is affected. To avoid side effects of general immunosuppression, local treatment by intraocular injection of anti-inflammatory or immunosuppressive drugs is desirable. Since we have shown previously that daily oral application of the small molecule DHODHinhibitor PP-001 is highly effective in suppressing experimental autoimmune uveitis in two different rat models (relapsing-remitting and chronic disease with neovascularizations) [2], we decided to investigate its therapeutic effect after intraocular injection. We have chosen our relapsing-remitting model to prevent relapses with a single intravitreal application of PP-001 after the primary uveitis attack had resolved, and we could observe a strongly reduced relapse rate with significantly less intense inflammation in the PP-001injected eyes within 6 days after treatment. This is the first time that the $\mathrm{DHODH}$-inhibitor PP-001 was proven to be active not only systemically, but also after local, intravitreal injection.

The short-term effect was thought to be due to the limited retention time of the therapeutic substance in ocular tissues after intraocular injection, which was subsequently confirmed by pharmacokinetic studies of PP-001 in rabbit eyes. The duration of the therapeutic effect exceeded the actual presence of PP-001 most likely due to the suppression of local autoreactive T cells, which remain in the eyes even after the resolution of ocular inflammation and can initiate recurrences of inflammation $[7,8]$. Tissue-resident $\mathrm{T}$ cells, which are reactivated later, or newly immigrating $\mathrm{T}$ cells would not be affected any more. This could explain the decreased effect on relapses of EAU later than 6 days post injection of PP-001.

After the first attack of uveitis had resolved to an activity score of 0.5 or less, some eyes showed increased inflammation at the time of injection of PP-001 in both groups. From the clinical point of view, this would be a typical scenario for the treatment in humans. Despite the increased activity, PP-001 still had a therapeutic effect. Overall, a single injection of aqueous formulated PP-001 was sufficient to achieve a local effect in the target tissue for several days. For long-lasting effects, the development of sustained release formulations is needed.

In rat models, PP-001 was shown to suppress in vitro proliferation of autoreactive $\mathrm{T}$ cells as well as secretion of certain inflammatory cytokines and chemokines [2]. Here, we demonstrate the suppression of proliferation of PHA-stimulated human PBL by increasing doses of PP001, yet not below the level of the (unstimulated) medium control, which indicates that PP-001 was not cytotoxic. Cytotoxicity for PBL was also excluded by daily determination of the viability of cells, which was not diminished even at high concentrations $(30 \mu \mathrm{M})$ of PP-001 (data not shown). The secretion of cytokines and chemokines of peripheral lymphocytes and RPE cells revealed that the levels of some factors secreted by $\mathrm{PBL}$ are strongly decreased with increasing doses of PP-001, while others remained unchanged or even increased. The latter is also arguing against general cytotoxicity of the small molecule. As observed with rat $\mathrm{T}$ cells [2], IFN- $\gamma$, IL-17, TNF- $\alpha$, and VEGF secretion was also strongly suppressed by PP-001 in human PBL. Since these cytokines play pivotal roles in inflammation in uveitis the inhibition of intraocular $\mathrm{T}$ cells by an immunomodulating agent like PP-001 would be of high therapeutic relevance [8-10].

Also, IL-2, IL-10, GM-CSF, and even the Th2 cytokine IL-13 were strongly downregulated. It is concluded from our data that the DHODH-inhibitor PP-001 has more influence on the cytokine secretion of lymphocytes than on monocytes/macrophages. The reason is most likely found in the higher demand for de novo pyrimidine synthesis during the activation of lymphocytes, conveying the specificity of PP-001 for T and B cells [3].

VEGF can be secreted by activated $\mathrm{T}$ cells and induce Th1 responses [11, 12], and we have previously shown that only rats with PDSAg-induced EAU developed chorioretinal neovascularization (CNV). Retinal destruction is usually severe in both types of EAU (monophasic and relapsing), and we observe CNV even in those eyes with only minor destruction of the retina [2], so we hypothesize that $\mathrm{CNV}$ in uveitis is induced by the VEGF-secretion of the intraocular PDSAg-specific $T$ cells rather than by ocular cells like RPE. This is supported by the fact that suppressing the $\mathrm{T}$ cells (and their VEGF secretion) by PP-001 was sufficient to prevent neovascularization in PDSAg-induced EAU [2].

The inhibition of VEGF secretion of human lymphocytes, but not of RPE cells by PP-001 might also be of therapeutic value for intraocular treatment in human uveitis cases with this sequel $[13,14]$. In patients, neovascularization has been described in cases of choroiditis or chorioretinitis, where treatment with VEGF-inhibitors is very successful [15]. It is assumed that, similar to the generation of lesions in AMD, local VEGF-production induces CNV. In addition, retinal neovascularization may develop in retinal inflammation like vasculitis without angiographic signs of ischemia or alterations of the RPE or choroid [13]. It may be speculated that in these patients, increased VEGF levels originate from inflammatory $\mathrm{T}$ cells located in the vitreous, retina, or the perivascular space and induce sprouting of new vessels out of the retinal vasculature. This would be consistent with the neovascularization observed in rat EAU.

Since VEGF is a therapeutic target for wet age-related macular degeneration (AMD) in patients, we were 
interested whether PP-001 could also suppress VEGF secretion by human retinal pigment epithelial cells, which are the major source of VEGF in AMD.

The anti-VEGF antibody bevacizumab had a marked effect on the secretion of several cytokines and especially on VEGF-secretion of RPE cells, while PP-001 had only marginal effects. We speculate that the decreased secretion of many cytokines by RPE cells could result from the depletion of VEGF, an important autocrine survival factor of RPE cells, by the VEGF-neutralizing antibody [16]. In contrast, secretion of IL-8/CXCL8 was increased by both PP-001 and bevacizumab. Like VEGF, IL-8/ CXCL8 also is an angiogenic [17] and anti-apoptotic survival factor for RPE cells [18], and both cytokines can induce neovascularization. VEGF even has the function of an autocrine survival factor for RPE cells $[19,20]$, and it is furthermore needed to maintain the vessels of the choriocapillaris [16, 21].

ARPE-19 cells express both VEGF receptors, VEGFR1 and VEGFR2, the latter is the major receptor to induce angiogenesis and to promote survival of RPE cells [22].

However, despite the positive effect of VEGF blockade on the leakiness of vessels and regression of neovascularization, severe damage like geographic atrophy and poor vision has been observed in AMD patients after extended treatment with anti-VEGF antibodies [23-25]. These adverse side effects would not be expected after PP-001 treatment, which is not affecting the RPE cells. Our data suggest that in patients with retinal vasculitis, PP-001 would specifically target intraocular $\mathrm{T}$ cells and their VEGF secretion and subsequent induction of neovascularization without disturbing the homeostasis of basal VEGF-secretion of RPE cells that is necessary to maintain the integrity of the choriocapillaris [21].

Regarding the effects of PP-001 on lymphocytes, our data suggest that the suppression of cytokine secretion is not a mere effect of suppression of proliferation and/ or the metabolism of the $\mathrm{T}$ cells by impeding pyrimidine synthesis, but there must be additional mechanisms, probably due to posttranscriptional regulation, as described for cytokines and chemokines by Fan et al. [26]. Increased mRNA stability might explain the increase of IL-8 secretion under PP-001 treatment in ARPE-19 cells.

During treatment with PP-001 and bevacizumab, IL-8 might serve as an autocrine survival factor for RPE, as described for endothelial cells [18, 27]. Moreover, interaction of IL-8/CXCL8 with its receptor CXCR2, both expressed by RPE cells, upregulates VEGF mRNA and protein, which results in autocrine activation of VEGFR2 $[28,29]$. Increased IL-8 production might therefore be a compensatory mechanism when VEGF secretion is inhibited.

\section{Conclusions}

The small molecule DHODH-inhibitor PP-001 is a useful therapeutic agent for intraocular application in autoimmune uveitis. PP-001 could suppress lymphocytes without affecting ocular tissues like the retinal pigment epithelium. In the latter case, the integrity of the choriocapillaris and the choroid will be preserved, in contrast to the current treatment of intraocular neovascularization with VEGF blockers. Sustained release formulations for intravitreal application of PP-001 are currently developed to prolong the efficacy of the treatment to several months post injection.

\section{Abbreviations}

CFA: Complete Freund's adjuvant; Cl: Confidence interval; CNV: Chorioretinal neovascularizations; DHODH: Dihydroorotate dehydrogenase; EAU: Experimental autoimmune uveitis; OD: Optical density; PBL: Peripheral blood lymphocytes; PHA: Phytohemagglutinin; RPE: Retinal pigment epithelium; VEGF: Vascular endothelial growth factor

\section{Acknowledgements}

Not applicable.

\section{Funding}

The study was supported by a grant from Panoptes Pharma GmbH (GW), the DFG grant PR1248/2-2, and EYEnovative Förderpreis 2012 (CSP). Panoptes Pharma is funded by LISA Seedfinancing administered by laws on behalf of the Austrian Ministry of Economy, Family and Youth (BMWFJ) and by grants of the Austrian Research Promotion Agency FFG.

Availability of data and materials

All data generated or analyzed during this study that are not included in this published article (e.g., data about the daily determination of viability of lymphocytes) can be obtained from the corresponding author.

\section{Authors' contributions}

SN performed the in vitro assays of human PBL, cultivation of RPE cells, and multiplex bead analysis. MD-M did the in vitro assays of human PBL and multiplex bead assay, animal experiments, and the design of the experiments; prepared the figures, and wrote the manuscript. CSP provided and established the primary human RPE cell cultures. SRT executed intravitreal injection of rat eyes and revised the manuscript. FO provided the therapeutic substance and revised the manuscript. SS is responsible for the pharmacokinetics of PP-001 in rabbit eyes and writing of the manuscript. GW provided idea and the design of the experiments, performed the experiments with RPE cells, evaluated the data, prepared the figures, and wrote the manuscript. All authors read and approved the final manuscript.

\section{Ethics approval and consent to participate}

The use of PBL from anonymized human donors with informed consent was approved by the ethics committee of the University Hospital, LMU Munich, Germany, and complied with the Declaration of Helsinki.

The isolation of primary human RPE cells from human cadaver eyes (anonymized donors) for scientific purposes was approved by the ethics committee of the Land Oberoesterreich. Proper consent and approval of the relatives was obtained, and the methods for securing human tissue were humane and complied with the Declaration of Helsinki. Human postmortem donor eyes were obtained from the Eye Bank of the Department of Ophthalmology at the Linz General Hospital (Linz, Austria).

\section{Consent for publication}

Not applicable.

\section{Competing interests}

The study was supported by a grant from Panoptes Pharma GmbH (GW). FO and SS are employees of Panoptes Pharma GmbH. The other authors do not have any competing interests regarding this project. 


\section{Publisher's Note}

Springer Nature remains neutral with regard to jurisdictional claims in published maps and institutional affiliations.

\begin{abstract}
Author details
'Section of Immunobiology, Department of Ophthalmology, University Hospital, LMU Munich, Mathildenstr. 8, 80336 Munich, Germany. ${ }^{2}$ Division Virus-associated carcinogenesis (F170), German Cancer Research Center (DKFZ), 69120 Heidelberg, Germany. ${ }^{3}$ Department of Ophthalmology, University Hospital, LMU Munich, Mathildenstr. 8, 80336 Munich, Germany. ${ }^{4}$ Panoptes Pharma GmbH, Reisnerstr. 34/1, 1030 Vienna, Austria.
\end{abstract}

Received: 10 November 2017 Accepted: 2 February 2018 Published online: 21 February 2018

\section{References}

1. Heiligenhaus A, Thurau S, Hennig M, Grajewski RS, Wildner G. Antiinflammatory treatment of uveitis with biologicals: new treatment options that reflect pathogenetic knowledge of the disease. Graefes Arch Clin Exp Ophthalmol. 2010;248(11):1531-51.

2. Diedrichs-Möhring M, Leban J, Strobl S, Obermayr F, Wildner G. A new small molecule for treating inflammation and chorioretinal neovascularization in relapsing-remitting and chronic experimental autoimmune uveitis: a new small molecule to treat monophasic and chronic EAU. Invest Ophthalmol Vis Sci. 2015;56(2):1147-57.

3. Fairbanks LD, Bofill M, Ruckemann K, Simmonds HA. Importance of ribonucleotide availability to proliferating T-lymphocytes from healthy humans: disproportionate expansion of pyrimidine pools and contrasting effects of de novo synthesis inhibitors. J Biol Chem. 1995;270(50):29682-9.

4. de Smet MD, Bitar G, Roberge FG, Gery I, Nussenblatt RB. Human S-antigen: presence of multiple immunogenic and immunopathogenic sites in the Lewis rat. J Autoimmun 1993;6(5):587-599.

5. Priglinger CS, Szober CM, Priglinger SG, Merl J, Euler KN, Kernt M, et al. Galectin-3 induces clustering of CD147 and integrin-beta1 transmembrane glycoprotein receptors on the RPE cell surface. PLoS One. 2013;8(7):e70011.

6. del Amo EM, Rimpelä A-K, Heikkinen E, Kari OK, Ramsay E, Lajunen T, et al. Pharmacokinetic aspects of retinal drug delivery. Prog Retin Eye Res. 2017;57(Supplement C):134-85.

7. von Toerne C, Sieg C, Kaufmann U, Diedrichs-Mohring M, Nelson PJ, Wildner G, Effector T. Cells driving monophasic vs. relapsing/remitting experimental autoimmune uveitis show unique pathway signatures. Mol Immunol. 2010;48(1-3):272-80.

8. Kaufmann U, Diedrichs-Mohring M, Wildner G. Dynamics of intraocular IFNgamma, IL-17 and IL-10-producing cell populations during relapsing and monophasic rat experimental autoimmune uveitis. PLoS One. 2012;7(11):e49008

9. Busch M, Bauer D, Hennig M, Wasmuth S, Thanos S, Heiligenhaus A. Effects of systemic and intravitreal TNF-a inhibition in experimental autoimmune uveoretinitis. Invest Ophthalmol Vis Sci. 2013;54(1):39-46.

10. Khera TK, Copland DA, Boldison J, Lait PJP, Szymkowski DE, Dick AD, et al. Tumour necrosis factor-mediated macrophage activation in the target organ is critical for clinical manifestation of uveitis. Clin Exp Immunol. 2012;168(2):165-77.

11. Mor F, Quintana FJ, Cohen IR. Angiogenesis-inflammation cross-talk: vascular endothelial growth factor is secreted by activated T cells and induces Th1 polarization. J Immunol. 2004;172(7):4618-23.

12. Basu A, Hoerning A, Datta D, Edelbauer M, Stack MP, Calzadilla K, et al. Cutting edge: vascular endothelial growth factor-mediated signaling in human CD45RO+ CD4+ T cells promotes Akt and ERK activation and costimulates IFN- $\gamma$ production. J Immunol. 2010;184(2):545-9.

13. Graham EM, Stanford MR, Shilling JS, Sanders MD. Neovascularisation associated with posterior uveitis. Br J Ophthalmol. 1987;71(11):826-33.

14. Neri P, Lettieri M, Fortuna C, Manoni M, Giovannini A. Inflammatory choroidal neovascularization. Middle East African Journal of Ophthalmology. 2009;16(4):245-51.

15. Battaglia Parodi M, lacono P, Verbraak FD, Bandello F. Antivascular endothelial growth factors for inflammatory chorioretinal disorders. Dev Ophthalmol. 2010;46:84-95.

16. Lee $\mathrm{S}$, Chen $\Pi$, Barber $\mathrm{CL}$, Jordan MC, Murdock J, Desai S, et al. Autocrine VEGF signaling is required for vascular homeostasis. Cell. 2007;130(4):691-703.

17. Brat DJ, Bellail AC, Van Meir EG. The role of interleukin-8 and its receptors in gliomagenesis and tumoral angiogenesis. Neuro-Oncology. 2005;7(2):122-33.
18. Li A, Dubey S, Varney ML, Dave BJ, Singh RK. IL-8 directly enhanced endothelial cell survival, proliferation, and matrix metalloproteinases production and regulated angiogenesis. J Immunol. 2003;170(6):3369-76.

19. Byeon SH, Lee SC, Choi SH, Lee H-K, Lee JH, Chu YK, et al. Vascular endothelial growth factor as an autocrine survival factor for retinal pigment epithelial cells under oxidative stress via the VEGF-R2/PI3K/Akt. Invest Ophthalmol Vis Sci. 2010;51(2):1190-7.

20. Fan J, Ponferrada VG, Sato T, Vemaraju S, Fruttiger M, Gerhardt $H$, et al. Crim1 maintains retinal vascular stability during development by regulating endothelial cell Vegfa autocrine signaling. Development (Cambridge, England). 2014;141(2):448-59.

21. Saint-Geniez M, Kurihara T, Sekiyama E, Maldonado AE, D'Amore PA. An essential role for RPE-derived soluble VEGF in the maintenance of the choriocapillaris. Proc Natl Acad Sci U S A. 2009;106(44):18751-6.

22. Spitzer MS, Wallenfels-Thilo B, Sierra A, Yoeruek E, Peters S, Henke-Fahle S, et al. Antiproliferative and cytotoxic properties of bevacizumab on different ocular cells. Br J Ophthalmol. 2006;90(10):1316-21.

23. Keck P, Hauser S, Krivi G, Sanzo K, Warren T, Feder J, et al. Vascular permeability factor, an endothelial cell mitogen related to PDGF. Science. 1989;246(4935):1309-12.

24. Penn JS, Madan A, Caldwell RB, Bartoli M, Caldwell RW, Hartnett ME. Vascular endothelial growth factor in eye disease. Prog Retin Eye Res. 2008;27(4):331-71

25. Deeg CA, Altmann F, Hauck SM, Schoeffmann S, Amann B, Stangassinger M, et al. Down-regulation of pigment epithelium-derived factor in uveitic lesion associates with focal vascular endothelial growth factor expression and breakdown of the blood-retinal barrier. Proteomics. 2007;7(9):1540-8.

26. Fan J, Heller NM, Gorospe M, Atasoy U, Stellato C. The role of posttranscriptional regulation in chemokine gene expression in inflammation and allergy. Eur Respir J. 2005;26(5):933-47.

27. Li A, Varney LM, Valasek J, Godfrey M, Dave JB, Singh KR. Autocrine role of interleukin-8 in induction of endothelial cell proliferation, survival, migration and MMP-2 production and angiogenesis. Angiogenesis. 2005;8(1):63-71.

28. Martin D, Galisteo R, Gutkind JS. CXCL8/IL8 stimulates vascular endothelial growth factor (VEGF) expression and the autocrine activation of VEGFR2 in endothelial cells by activating NFKB through the CBM (Carma3/Bcl10/Malt1) complex. J Biol Chem. 2009:284(10):6038-42.

29. Palma-Nicolas JP, Lopez E, Lopez-Colome AM. Thrombin stimulates RPE cell motility by PKC-zeta- and NF-kappaB-dependent gene expression of MCP-1 and CINC-1/GRO chemokines. J Cell Biochem. 2010;110(4):948-67.

\section{Submit your next manuscript to BioMed Central and we will help you at every step:}

- We accept pre-submission inquiries

- Our selector tool helps you to find the most relevant journal

- We provide round the clock customer support

- Convenient online submission

- Thorough peer review

- Inclusion in PubMed and all major indexing services

- Maximum visibility for your research

Submit your manuscript at www.biomedcentral.com/submit
) Biomed Central 